\title{
Cold and ultracold NH-NH collisions in magnetic fields
}

\author{
Liesbeth M. C. Janssen, ${ }^{1}$ Piotr S. Żuchowski, ${ }^{2}$ Ad van der Avoird, ${ }^{1}$ Gerrit C. Groenenboom, ${ }^{1, *}$ and Jeremy M. Hutson ${ }^{2, \dagger}$ \\ ${ }^{1}$ Radboud University Nijmegen, Institute for Molecules and Materials, Heyendaalseweg 135, 6525 AJ Nijmegen, The Netherlands \\ ${ }^{2}$ Department of Chemistry, Durham University, South Road, Durham, DH1 3LE, United Kingdom
}

(Received 13 December 2010; published 28 February 2011)

\begin{abstract}
Elastic and spin-changing inelastic collision cross sections are presented for cold and ultracold magnetically trapped $\mathrm{NH}$. The cross sections are obtained from coupled-channel scattering calculations as a function of energy and magnetic field. We specifically investigate the influence of the intramolecular spin-spin, spin-rotation, and intermolecular magnetic dipole coupling on the collision dynamics. It is shown that ${ }^{15} \mathrm{NH}$ is a very suitable candidate for evaporative cooling experiments. The dominant trap-loss mechanism in the ultracold regime originates from the intermolecular dipolar coupling term. At higher energies and fields, intramolecular spin-spin coupling becomes increasingly important. Our qualitative results and conclusions are fairly independent of the exact form of the potential and of the size of the channel basis set.
\end{abstract}

DOI: 10.1103/PhysRevA.83.022713

PACS number(s): 34.50.Cx, 37.10.Mn, 37.10.Pq

\section{INTRODUCTION}

Cold $(T<1 \mathrm{~K})$ and ultracold $(T<1 \mathrm{mK})$ molecules have attracted great interest in recent years due to their potential applications in condensed matter physics [1], quantum computing [2,3], high-precision spectroscopy [4-6], and physical chemistry [7-11]. Experimental methods for producing ultracold molecular samples include photoassociation [12] and Feshbach association [13], in which molecules are formed by linking two ultracold atoms. These indirect cooling techniques are, however, limited to molecules consisting of two (alkali metal) atoms that can be efficiently precooled to ultralow temperatures. Direct cooling methods such as buffer-gas cooling [14] and Stark deceleration [15], in which molecules are cooled from room temperature, are applicable to a much wider range of species.

The NH radical is an excellent candidate for direct cooling experiments, due to its relatively large magnetic and electric dipole moments. Ground-state $\mathrm{NH}\left(X^{3} \Sigma^{-}\right)$has been successfully cooled with a helium buffer gas and confined in a magnetic trap [10,16-18]. Metastable $\mathrm{NH}\left(a^{1} \Delta\right)$ has been Stark decelerated and trapped in an electrostatic field [19]. Furthermore, an optical pumping scheme has been developed to transfer the Stark-decelerated molecules to the electronic ground state and subsequently accumulate them in a superimposed magnetic trap [20].

At present, direct cooling experiments for $\mathrm{NH}$ have achieved temperatures of a few hundred millikelvins. One of the key challenges is to produce molecules in the ultracold regime, which requires a second-stage cooling method. Examples of such methods include sympathetic cooling with an ultracold co-trapped species [21-25] and evaporative cooling. The latter approach relies on elastic $\mathrm{NH}+\mathrm{NH}$ collisions that thermalize the gas as the magnetic trap depth is slowly decreased. Inelastic collisions, however, can induce Zeeman relaxation and consequently lead to trap loss. Therefore, in

\footnotetext{
*gerritg@theochem.ru.nl

†j.m.hutson@durham.ac.uk
}

order for evaporative cooling to work, elastic collisions should be much more efficient than inelastic ones, typically by a few orders of magnitude [26]. Evaporative cooling has already been achieved for buffer-gas-cooled metastable helium [27], indicating that the densities in buffer-gas cooling experiments are high enough to obtain a sufficiently high elastic collision rate. This also provides hope for other buffer-gas-cooled species such as NH.

A collision complex consisting of two magnetically trapped NH molecules is in the $\left|S=2, M_{S}=2\right\rangle$ state, with $S$ denoting the total spin and $M_{S}$ its laboratory-frame projection. Inelastic $\mathrm{NH}+\mathrm{NH}$ collisions can change the $M_{S}$ quantum number and/or the total spin of the dimer. If the total spin is flipped, the complex undergoes a transition from the quintet state to the chemically reactive singlet or triplet states [28-30]. Although unfavorable for evaporative cooling, the latter two spin states may be relevant in the context of cold controlled chemistry [11].

Previous theoretical work by Kajita [31] and Janssen et al. [32] has shown that $\mathrm{NH}$ is a promising candidate for molecular evaporative cooling, particularly the bosonic ${ }^{15} \mathrm{NH}$ isotopologue. Fermionic ${ }^{14} \mathrm{NH}$ may also be cooled into the $\mu \mathrm{K}$ regime either by applying an electric field [31] or by preparing the molecules in different hyperfine states [32]. It must be noted, however, that the results of Kajita were obtained from approximate analytical methods in which only the electric dipole-dipole and magnetic dipole-dipole interactions were included. The work of Ref. [32] involved rigorous quantum scattering calculations on a full four-dimensional potentialenergy surface, but focused only on $\mathrm{NH}+\mathrm{NH}$ collisions in zero field.

In this paper, we consider collisions between ${ }^{15} \mathrm{NH}$ molecules in the presence of an external magnetic field. We present full quantum scattering calculations on an accurate $a b$ initio quintet potential to investigate the collision dynamics at low and ultralow temperatures. Intramolecular spin-spin coupling, spin-rotation, and intermolecular magnetic dipole (spin-spin) coupling are explicitly included in the calculations. We identify the dominant trap-loss mechanisms and provide a detailed discussion of the dynamics. 


\section{CALCUlations}

\section{A. Theory}

We consider elastic and inelastic collisions between two identical $\mathrm{NH}\left(X^{3} \Sigma^{-}\right)$molecules in the presence of a magnetic field. Since bosonic ${ }^{15} \mathrm{NH}$ is more suitable for evaporative cooling that fermionic ${ }^{14} \mathrm{NH}$, we focus only on the ${ }^{15} \mathrm{NH}$ isotopologue. We treat the colliding molecules as rigid rotors. The coordinate system is defined in a space-fixed frame, with $\boldsymbol{R} \equiv(R, \Omega)$ and $\Omega=(\Phi, \Theta)$ denoting the intermolecular vector between the centers of mass of the molecules. The coordinates $\omega_{A}=\left(\theta_{A}, \phi_{A}\right)$ and $\omega_{B}=\left(\theta_{B}, \phi_{B}\right)$ are the polar angles of monomers $A$ and $B$, respectively. We neglect hyperfine structure and assume that both molecules are in their nuclearspin stretched states $\left|I, M_{I}=I\right\rangle$, with $I=I_{\mathrm{N}}+I_{\mathrm{H}}=1$ denoting the maximum total nuclear spin and $M_{I}$ its laboratory-frame projection. The scattering Hamiltonian is then given by

$$
\begin{aligned}
\hat{H}= & -\frac{\hbar^{2}}{2 \mu R} \frac{\partial^{2}}{\partial R^{2}} R+\frac{\hat{L}^{2}}{2 \mu R^{2}}+V_{S}\left(\boldsymbol{R}, \omega_{A}, \omega_{B}\right) \\
& +V_{\text {magn.dip }}\left(\boldsymbol{R}, \hat{\boldsymbol{S}}_{A}, \hat{\boldsymbol{S}}_{B}\right)+\hat{H}_{A}+\hat{H}_{B},
\end{aligned}
$$

where $\mu$ is the reduced mass of the complex, $\hat{L}^{2}$ is the angular momentum operator associated with end-over-end rotation of the vector $\boldsymbol{R}, V_{S}\left(\boldsymbol{R}, \omega_{A}, \omega_{B}\right)$ is the potential-energy surface for total spin $S, V_{\text {magn.dip }}\left(\boldsymbol{R}, \hat{\boldsymbol{S}}_{A}, \hat{\boldsymbol{S}}_{B}\right)$ is the intermolecular magnetic dipole interaction, and $\hat{H}_{A}$ and $\hat{H}_{B}$ are the Hamiltonians of the two monomers. The intermolecular magnetic dipole term is given by

$$
\begin{aligned}
& V_{\text {magn.dip }}\left(\boldsymbol{R}, \hat{\boldsymbol{S}}_{A}, \hat{\boldsymbol{S}}_{B}\right) \\
& \quad=-\sqrt{6} g_{S}^{2} \mu_{\mathrm{B}}^{2} \frac{\alpha^{2}}{R^{3}} \sum_{q}(-1)^{q} C_{2,-q}(\Omega)\left[\hat{\boldsymbol{S}}_{A} \otimes \hat{\boldsymbol{S}}_{B}\right]_{q}^{(2)}
\end{aligned}
$$

where $g_{S} \approx 2.0023$ is the electron $g$ factor, $\mu_{\mathrm{B}}$ is the Bohr magneton, $\alpha$ is the fine-structure constant, and the factor in square brackets is the tensor product of the monomer spin operators $\hat{\boldsymbol{S}}_{A}$ and $\hat{\boldsymbol{S}}_{B}$. The monomer operators $\hat{H}_{i}(i=A, B)$ each contain a rotation, spin-rotation, intramolecular spin-spin, and Zeeman term,

$$
\begin{aligned}
\hat{H}_{i}= & B_{0} \hat{N}_{i}^{2}+\gamma \hat{\boldsymbol{N}}_{i} \cdot \hat{\boldsymbol{S}}_{i}+\frac{2}{3} \sqrt{6} \lambda_{\mathrm{SS}} \\
& \times \sum_{q}(-1)^{q} C_{2,-q}\left(\omega_{i}\right)\left[\hat{\boldsymbol{S}}_{i} \otimes \hat{\boldsymbol{S}}_{i}\right]_{q}^{(2)}+g_{S} \mu_{\mathrm{B}} \boldsymbol{B} \cdot \hat{\boldsymbol{S}}_{i},
\end{aligned}
$$

where $\hat{\boldsymbol{N}}_{i}$ is the operator for the rotational angular momentum of monomer $i$ and $\boldsymbol{B}$ is the magnetic field vector. The numerical values for the rotational, spin-rotation, and spin-spin constants of ${ }^{15} \mathrm{NH}$ can be obtained from those of ${ }^{14} \mathrm{NH}$ [33] using isotope scaling (see, e.g., p. 239 of Ref. [34]): $B_{0}=16.270340 \mathrm{~cm}^{-1}$, $\gamma=-0.05460 \mathrm{~cm}^{-1}$, and $\lambda_{\mathrm{SS}}=0.91989 \mathrm{~cm}^{-1}$.

We will assume that all three spin states of the complex are described by the nonreactive quintet surface, i.e., $V_{S}\left(\boldsymbol{R}, \omega_{A}, \omega_{B}\right) \equiv V_{2}\left(\boldsymbol{R}, \omega_{A}, \omega_{B}\right)$. Field-free calculations have shown that this assumption is reasonable [32]. The $S=2$ surface is taken from Ref. [29]. The potential is expanded in coupled spherical harmonics $Y_{L, M}$ [35],

$$
\begin{aligned}
& V\left(\boldsymbol{R}, \omega_{A}, \omega_{B}\right) \\
& =\sum_{L_{A}, L_{B}, L_{A B}} v_{L_{A}, L_{B}, L_{A B}}(R) A_{L_{A}, L_{B}, L_{A B}}\left(\Omega, \omega_{A}, \omega_{B}\right), \\
& A_{L_{A}, L_{B}, L_{A B}}\left(\Omega, \omega_{A}, \omega_{B}\right) \\
& =\sum_{\substack{M_{A}, M_{B}, M_{A B} \\
\quad}}\left\langle L_{A} M_{A} L_{B} M_{B} \mid L_{A B} M_{A B}\right\rangle \\
& \quad \times Y_{L_{A}, M_{A}}\left(\omega_{A}\right) Y_{L_{B}, M_{B}}\left(\omega_{B}\right) Y_{L_{A B}, M_{A B}}^{*}(\Omega),
\end{aligned}
$$

where $\left\langle L_{A} M_{A} L_{B} M_{B} \mid L_{A B} M_{A B}\right\rangle$ is a Clebsch-Gordan coefficient and the superscript $*$ denotes complex conjugation. The subscript $S=2$ has been omitted for brevity. As noted previously in Ref. [32], the $v_{L_{A}, L_{B}, L_{A B}}(R)$ expansion coefficients of Ref. [29] must be multiplied by a factor of $(-1)^{L_{A}-L_{B}}(4 \pi)^{-3 / 2}\left(2 L_{A B}+1\right)\left[\left(2 L_{A}+1\right)\left(2 L_{B}+1\right)\right]^{1 / 2}$ to obtain the potential in the form of Eq. (4).

We expand the wave function in a space-fixed uncoupled basis introduced in Ref. [36]. The angular functions are written as products of the eigenfunctions of $\hat{N}_{i}^{2}, \hat{N}_{i_{z}}, \hat{S}_{i}^{2}, \hat{S}_{i_{z}}, \hat{L}^{2}$, and $\hat{L}_{z}$,

$$
\begin{aligned}
& \left|N_{A} M_{N_{A}}\right\rangle\left|S_{A} M_{S_{A}}\right\rangle\left|N_{B} M_{N_{B}}\right\rangle\left|S_{B} M_{S_{B}}\right\rangle\left|L M_{L}\right\rangle \\
& \quad \equiv\left|\gamma_{A} \gamma_{B}\right\rangle\left|L M_{L}\right\rangle,
\end{aligned}
$$

with $N_{A}$ and $N_{B}$ ranging from 0 to $N_{\max }$ and $L=0, \ldots, L_{\max }$. The matrix elements of the scattering Hamiltonian [Eq. (1)] in the basis of Eq. (6) have all been given by Krems and Dalgarno [36]. Note that the factor of $g_{S}^{2} \mu_{\mathrm{B}}^{2} \approx 1.0023 e^{2} \hbar^{2} / m_{e}^{2}$ has been neglected in their expression for the magnetic dipole interaction. Furthermore, it should be taken into account that our potential expansion [Eq. (4)] differs by a factor of $(-1)^{L_{A}-L_{B}}(4 \pi)^{3 / 2}\left(2 L_{A B}+1\right)^{1 / 2}$ from the expansion used in Ref. [36].

Since the monomers are identical, we can exploit the permutation symmetry of the system to minimize the computational cost. Following Ref. [37], we employ a normalized, symmetrized basis of the form

$$
\begin{aligned}
\left|\phi_{\gamma_{A} \gamma_{B} L M_{L}}^{\eta}\right\rangle= & \frac{1}{\left[2\left(1+\delta_{\gamma_{A} \gamma_{B}}\right)\right]^{1 / 2}}\left[\left|\gamma_{A} \gamma_{B}\right\rangle\left|L M_{L}\right\rangle\right. \\
& \left.+\eta(-1)^{L}\left|\gamma_{B} \gamma_{A}\right\rangle\left|L M_{L}\right\rangle\right],
\end{aligned}
$$

with $\eta= \pm 1$ defining the symmetry of the wave function with respect to molecular interchange. It is sufficient to restrict the basis to a well-ordered set of molecular states, i.e., $\gamma_{A} \geqslant \gamma_{B}$. Finally, we note that the parity, $\epsilon=(-1)^{N_{A}+N_{B}+L}$, and the total angular momentum projection quantum number, $\mathcal{M}=M_{N_{A}}+M_{S_{A}}+M_{N_{B}}+M_{S_{B}}+M_{L}$, are also conserved during collision. Thus, the scattering calculations may be performed for a single value of $\mathcal{M}, \eta$, and $\epsilon$. Here we consider only collisions between bosonic ${ }^{15} \mathrm{NH}$ molecules in their spin-stretched and rotational ground state $\left(N_{A}=N_{B}=0\right)$, for which we have $\eta=+1$ and $\epsilon=+1$. Note that the first excited rotational state has an energy of $\approx 32 \mathrm{~cm}^{-1}(46 \mathrm{~K})$ and is therefore inaccessible at the energies considered in this work.

We solve the coupled equations using the hybrid logderivative method of Alexander and Manolopoulos [38], which uses a fixed-step-size log-derivative propagator at 
short range and a variable-step-size Airy propagator at long range. Matching to asymptotic boundary conditions yields the scattering $S$ matrices, from which the cross sections can be readily obtained. We note that, due to the intramolecular spin-rotation and spin-spin couplings, the basis functions of Eq. (7) are not exact eigenfunctions of the asymptotic Hamiltonian $\hat{H}_{A}+\hat{H}_{B}$, while the $S$ matrices must be constructed in terms of these eigenfunctions. As detailed in Refs. [36] and [37], an additional basis transformation of the log-derivative matrix is therefore required before matching to the asymptotic boundary conditions. We denote the symmetrized channel eigenfunctions as $\left|\phi_{\bar{\gamma}_{A} \bar{\gamma}_{B} L M_{L}}^{\eta}\right\rangle$, with $\left|\bar{\gamma}_{A} \bar{\gamma}_{B}\right\rangle \equiv$ $\left|\left(\bar{N}_{A}, S_{A}\right) J_{A}, M_{J_{A}}\right\rangle\left|\left(\bar{N}_{B}, S_{B}\right) J_{B}, M_{J_{B}}\right\rangle$ defining the molecular eigenstates. Here $\bar{N}_{i}$ is used instead of $N_{i}$, because $N_{i}$ is strictly not an exact quantum number due to the intramolecular spin-spin coupling. However, the intramolecular coupling is relatively weak and $N_{i}, M_{N_{i}}$, and $M_{S_{i}}$ may be treated as approximately good quantum numbers. Specifically, for the ${ }^{15} \mathrm{NH}$ rotational ground state, the magnetically trapped $\mid J_{i}=$ $\left.1, M_{J_{i}}=1\right\rangle$ component contains $99.992 \%$ of $\mid N_{i}=0, M_{N_{i}}=$ $\left.0, S_{i}=1, M_{S_{i}}=1\right\rangle$ for all fields considered in this work.

The cross sections at total energy $E$ are calculated using the expression [37]

$$
\begin{aligned}
& \sigma_{\bar{\gamma}_{A} \bar{\gamma}_{B} \rightarrow \bar{\gamma}_{A}^{\prime} \bar{\gamma}_{B}^{\prime}}^{\eta}(E) \\
& \quad=\frac{\pi\left(1+\delta_{\bar{\gamma}_{A} \bar{\gamma}_{B}}\right)}{k_{\bar{\gamma}_{A} \bar{\gamma}_{B}}^{2}} \sum_{L, M_{L}} \sum_{L^{\prime}, M_{L}^{\prime}}\left|T_{\bar{\gamma}_{A} \bar{\gamma}_{B} L M_{L} ; \bar{\gamma}_{A}^{\prime} \bar{\gamma}_{B}^{\prime} L^{\prime} M_{L}^{\prime}}^{\eta}(E)\right|^{2},
\end{aligned}
$$

where $k_{\bar{\gamma}_{A} \bar{\gamma}_{B}}$ is the wave number for the incident channel, $k_{\bar{\gamma}_{A} \bar{\gamma}_{B}}^{2}=2 \mu\left(E-\epsilon_{\bar{\gamma}_{A}}-\epsilon_{\bar{\gamma}_{B}}\right) / \hbar^{2}, \epsilon_{\bar{\gamma}_{A}}+\epsilon_{\bar{\gamma}_{B}}$ is the corresponding channel energy, and the $T$-matrix elements are defined as $T_{\bar{\gamma}_{A} \bar{\gamma}_{B} L M_{L} ; \bar{\gamma}_{A}^{\prime} \bar{\gamma}_{B}^{\prime} L^{\prime} M_{L}^{\prime}}^{\eta}=\delta_{\bar{\gamma}_{A} \bar{\gamma}_{A}^{\prime}} \delta_{\bar{\gamma}_{B} \bar{\gamma}_{B}^{\prime}} \delta_{L L^{\prime}} \delta_{M_{L} M_{L}^{\prime}}-$ $S_{\bar{\gamma}_{A} \bar{\gamma}_{B} L M_{L} ; \bar{\gamma}_{A}^{\prime} \bar{\gamma}_{B}^{\prime} L^{\prime} M_{L}^{\prime}}$

\section{B. Computational details}

We performed the scattering calculations for different magnetic fields using the MOLSCAT package [39,40], extended to handle the basis set of Eq. (7). The log-derivative propagation was carried out on a radial grid ranging from $4.5 a_{0}$ to $15 a_{0}$ in steps of $0.02 a_{0}$. The Airy propagation ranged from $15 a_{0}$ to $50000 a_{0}$. We included basis functions up to $N_{\max }=2$ and $L_{\max }=6$, yielding a maximum number of 1038 channels for a single calculation $(\mathcal{M}=0)$. The expansion of the quintet potential-energy surface was truncated at $L_{A}=L_{B}=6$. As mentioned in Sec. II A, the chemically reactive singlet and triplet potentials were not included in the calculations.

\section{RESULTS AND DISCUSSION}

\section{A. Adiabatic potential-energy curves}

Before discussing the cross sections, we first consider the adiabatic potential curves. These are obtained by diagonalizing the interaction matrix at fixed $R$ over a grid of $R$ values and subsequently connecting the corresponding eigenvalues. Asymptotically, these adiabatic curves correlate to the molecular eigenstates $\bar{\gamma}_{A}$ and $\bar{\gamma}_{B}$. Figure 1 shows the long range of the lowest adiabats for $\mathcal{M}=2$ and $L_{\max }=4$ at a magnetic field of $0.1 \mathrm{G}$. We present only the curves with exchange symmetry

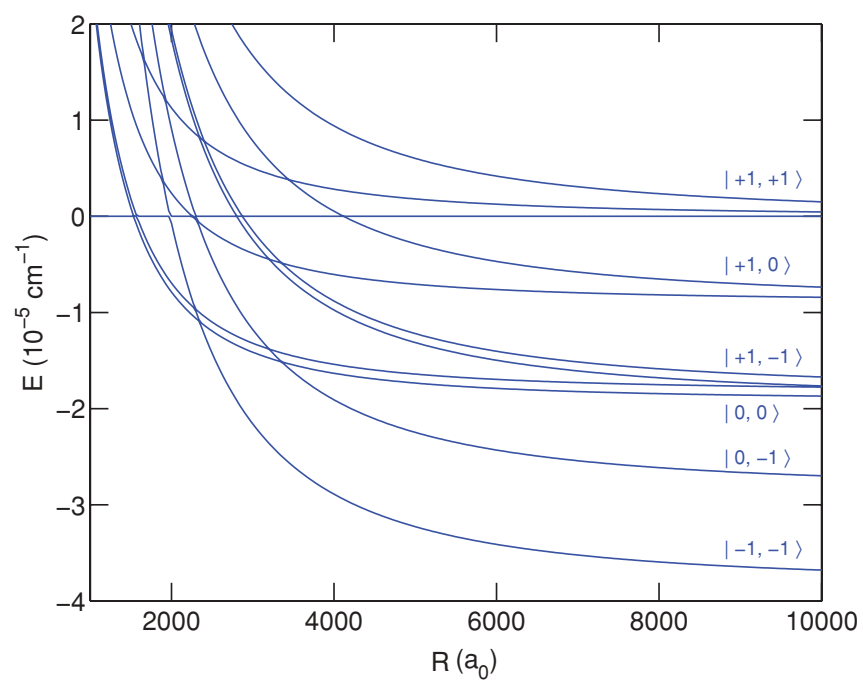

FIG. 1. (Color online) Lowest adiabatic potential curves for ${ }^{15} \mathrm{NH}-{ }^{15} \mathrm{NH}$, calculated for $L_{\max }=4$ and $\mathcal{M}=2$ at a magnetic field of $0.1 \mathrm{G}$. The adiabats are plotted as a function of $R$ in units of the Bohr radius $a_{0}$. The molecular eigenstates are labeled by $\left|M_{J_{A}}, M_{J_{B}}\right\rangle$ and refer to the well-ordered states $\left|\left(N_{A}=0\right) J_{A}=1, M_{J_{A}}\right\rangle \mid\left(N_{B}=\right.$ 0) $\left.J_{B}=1, M_{J_{B}}\right\rangle$.

$\eta=+1$ and parity $\epsilon=+1$, for which $s$-wave scattering in the incident spin-stretched channel is allowed. The asymptotic energy splittings between the different molecular states originate from the Zeeman term $g_{S} \mu_{\mathrm{B}} B\left(M_{S_{A}}+M_{S_{B}}\right)$. For $\mathcal{M}=2$ and $L_{\max }=4$, the magnetically trapped state with $\mid M_{J_{A}}=$ $\left.M_{J_{B}}=1\right\rangle$ has partial-wave contributions of $L=0,2$, and 4, as can be seen in Fig. 1. The adiabats correlating with $\mid M_{J_{A}}=$ $\left.1, M_{J_{B}}=0\right\rangle,\left|M_{J_{A}}=1, M_{J_{B}}=-1\right\rangle$, and $\left|M_{J_{A}}=0, M_{J_{B}}=0\right\rangle$ have $L=2$ and 4 centrifugal barriers, and the adiabats for $\left|M_{J_{A}}=0, M_{J_{B}}=-1\right\rangle$ and $\left|M_{J_{A}}=-1, M_{J_{B}}=-1\right\rangle$ contain only the $L=4$ partial wave. For the $\left|M_{J_{A}}=1, M_{J_{B}}=-1\right\rangle$ and $\left|M_{J_{A}}=0, M_{J_{B}}=0\right\rangle$ states, which have identical Zeeman shifts, the degeneracy is further lifted by the intramolecular spin-spin coupling.

It can be seen that several curve crossings occur in the region between $R \approx 1500 a_{0}$ and $4500 a_{0}$. If we neglect the small energy shifts due to the intramolecular coupling, the crossing points $R_{c}$ are simply given by

$$
g_{S} \mu_{\mathrm{B}} B \Delta M_{S}=\frac{\hbar^{2}\left[L_{f}\left(L_{f}+1\right)-L_{i}\left(L_{i}+1\right)\right]}{2 \mu R_{c}^{2}},
$$

where $L_{i}$ and $L_{f}$ denote the values of $L$ for the adiabats correlating to the incoming and outgoing channels, respectively, and $\Delta M_{S}=M_{S_{A}}^{(i)}+M_{S_{B}}^{(i)}-M_{S_{A}}^{(f)}-M_{S_{B}}^{(f)}$. The corresponding energies at which the crossings occur are given by $E_{c}=\hbar^{2} L_{i}\left(L_{i}+1\right) /\left(2 \mu R_{c}^{2}\right)$, defined relative to the threshold of the incident channel. We find that several of these crossings are narrowly avoided due to the presence of the intermolecular magnetic dipole interaction. Inspection of Eq. (30) of Ref. [36] or Eq. (A2) of Ref. [41] shows that, e.g., the $s$-wave incident channel with $\mid M_{S_{A}}=1, M_{S_{B}}=1, L=$ $0\rangle$ is directly coupled with $\left|M_{S_{A}}=1, M_{S_{B}}=0, L=2\right\rangle$ and $\left|M_{S_{A}}=0, M_{S_{B}}=0, L=2\right\rangle$ via the magnetic dipole term, giving rise to the corresponding avoided crossings. We note, however, that $V_{\text {magn.dip }}$ contains a second-rank tensor in $\Omega$ and 
can therefore directly couple channels only if $\left|L_{i}-L_{f}\right| \leqslant 2$. The spin operators $\hat{\boldsymbol{S}}_{A}$ and $\hat{\boldsymbol{S}}_{B}$ contained in $V_{\text {magn.dip }}$ are first-rank tensors and, consequently, $M_{S_{A}}$ and $M_{S_{B}}$ may each differ at most by 1 . Thus, not all crossings are avoided. To take proper account of these avoided crossings and account correctly for the magnetic dipole coupling in the ultracold regime, it is essential that the radial grid used in the calculations extends beyond the outermost $R_{c}$ value. The influence of the avoided crossings on the collision cross sections is discussed in detail in the following section.

\section{B. Cross sections}

Figure 2 shows the total elastic and $M_{J}$-changing inelastic cross sections for two magnetically trapped ${ }^{15} \mathrm{NH}$ molecules $\left(M_{J_{A}}=M_{J_{B}}=1\right)$ as a function of collision energy. The cross sections as a function of magnetic field are given in Fig. 3. We find that the elastic cross sections are significantly larger than the inelastic ones over a wide range of energies and magnetic fields, suggesting that evaporative cooling of ${ }^{15} \mathrm{NH}$ is likely to be successful. It can also be seen that, in the ultracold regime, the total inelastic cross section decreases dramatically if the magnetic field strength is reduced. Thus, once the cooling process has started in the millikelvin regime at relatively high magnetic field and continues toward lower energies as the magnetic trap depth is decreased, the ratio between elastic and $M_{J}$-changing cross sections will remain very favorable for evaporative cooling to take place.

In order to identify the main trap-loss mechanism, we have also performed scattering calculations where two of the three spin-dependent coupling terms are set to zero. The results for $\mathcal{M}=2$ are shown in Figs. 4 and 5 . We have verified that the $\mathcal{M}=2$ cross sections, which include contributions from $s$-wave collisions in the incident channel, are dominant at the energies and fields considered in this work. It can be seen that the intermolecular spin-spin interaction $\left(V_{\text {magn.dip }}\right)$ gives the largest contribution to the inelastic cross section over a

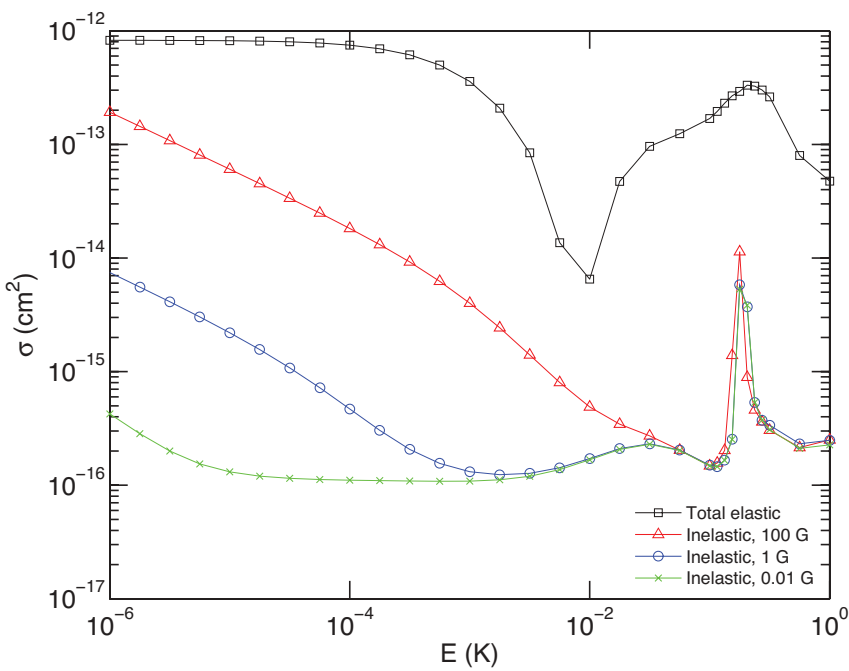

FIG. 2. (Color online) Elastic and inelastic $M_{J}$-changing cross sections for magnetically trapped ${ }^{15} \mathrm{NH}$ as a function of collision energy for various magnetic fields. The elastic cross sections are the same for all three magnetic field strengths.

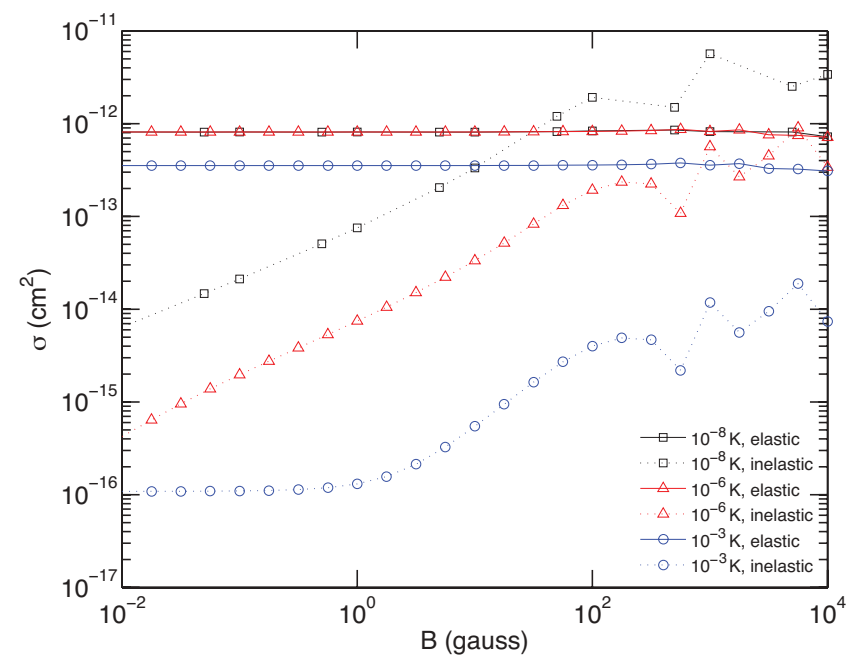

FIG. 3. (Color online) Elastic and inelastic $M_{J}$-changing cross sections for magnetically trapped ${ }^{15} \mathrm{NH}$ as a function of magnetic field for various collision energies.

broad range of energies and field strengths, most notably in the ultracold regime. This is also the case for cold and ultracold $\mathrm{N}+\mathrm{NH}$ collisions [24,25]. At higher collision energies and fields, however, the intramolecular coupling terms become increasingly important. In particular the intramolecular spinspin term causes significant trap loss above $E \approx 10^{-2} \mathrm{~K}$ and $B \approx 100 \mathrm{G}$. Spin-rotation coupling, which vanishes for pure $N_{A}=N_{B}=0$ states, has only a very small effect on the total cross section. This is consistent with previous work on the $\mathrm{He}-\mathrm{NH}\left({ }^{3} \Sigma^{-}\right)$system $[16,36,42]$.

The importance of the intermolecular magnetic dipole interaction is most easily understood by considering the adiabatic potential curves. As discussed in Sec. III A, the avoided crossings between the $s$-wave incoming channel

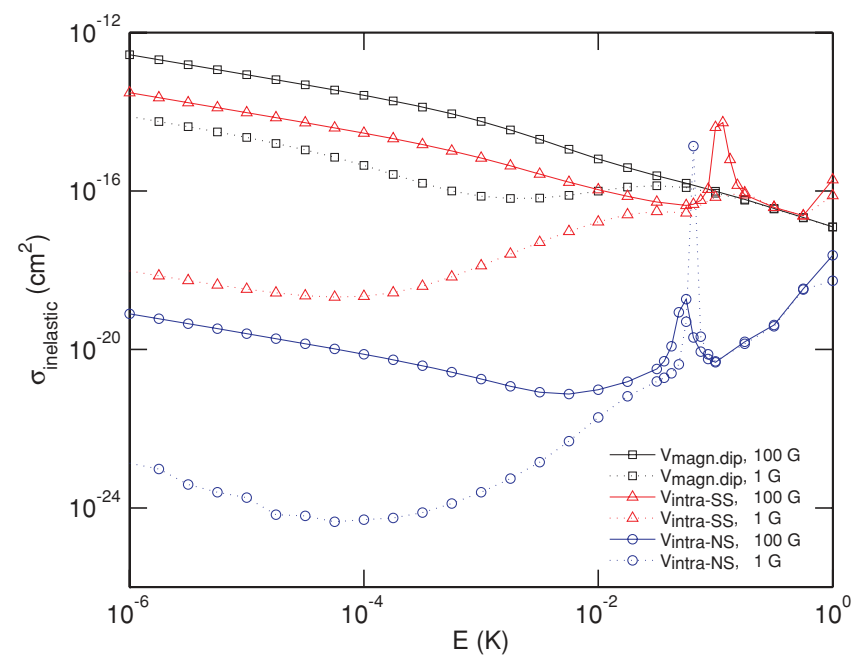

FIG. 4. (Color online) Inelastic $M_{J}$-changing cross sections for $\mathcal{M}=2$ as a function of collision energy at 1 and $100 \mathrm{G}$. The different curves correspond to calculations with only the intermolecular magnetic dipolar coupling ( $\left.V_{\text {magn.dip }}\right)$, the intramolecular spin-spin coupling $\left(V_{\text {intra-ss }}\right)$, or the intramolecular spin-rotation coupling $\left(V_{\text {intra-NS }}\right)$ included in $\hat{H}$. 


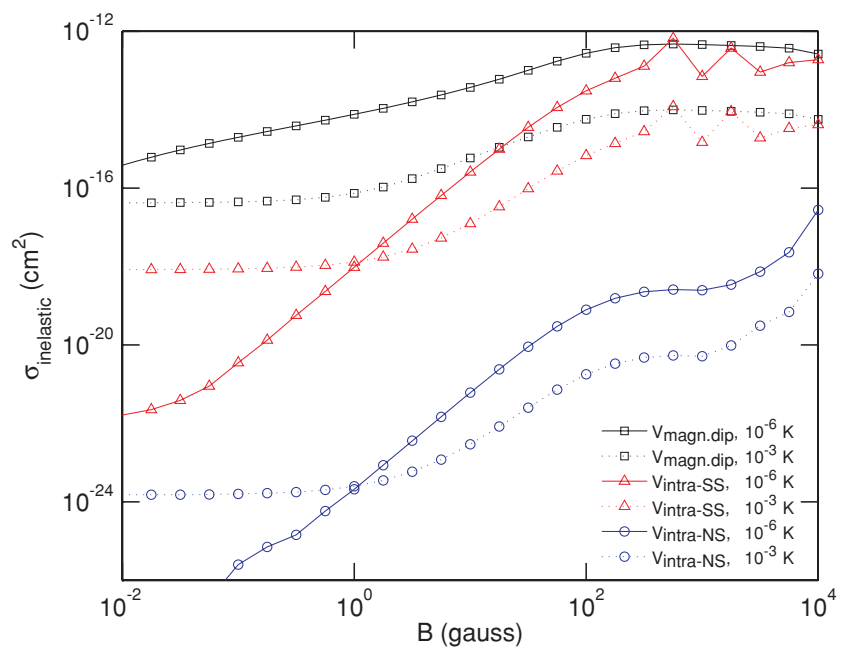

FIG. 5. (Color online) Inelastic $M_{J}$-changing cross sections for $\mathcal{M}=2$ as a function of magnetic field at $10^{-6} \mathrm{~K}$ and $10^{-3} \mathrm{~K}$. The different curves are obtained from scattering calculations with only the intermolecular magnetic dipolar coupling $\left(V_{\text {magn.dip }}\right)$, the intramolecular spin-spin coupling $\left(V_{\text {intra-sS }}\right)$, or the intramolecular spin-rotation coupling $\left(V_{\text {intra-NS }}\right)$ switched on.

$\left|M_{J_{A}}=1, M_{J_{B}}=1, L=0\right\rangle$ and the $\mid M_{S_{A}}=1, M_{S_{B}}=0, L=$ 2) and $\left|M_{S_{A}}=0, M_{S_{B}}=0, L=2\right\rangle$ outgoing channels all occur at very long range for small to moderate field strengths. Consequently, the spin-flip induced by $V_{\text {magn.dip }}$ can take place without having to overcome the $d$-wave barrier in the outgoing channels, and hence this process dominates the inelastic cross section in the ultracold regime. We also emphasize that, if the avoided-crossing points $R_{c}$ fall outside the scattering propagation region, i.e., if the radial grid is chosen too small, the inelastic cross sections are similar to the case where the intermolecular magnetic dipole term is switched off completely. This confirms that the spin-flip due to $V_{\text {magn.dip }}$ indeed takes place at long range, or more specifically at $R \approx R_{c}$.

Kajita [31] has shown that, in the Born approximation, the inelastic cross section $\sigma_{i \rightarrow f}$ for ${ }^{15} \mathrm{NH}\left(L_{i}=\right.$ $0, L_{f}=2$ ) caused by the magnetic dipole interaction should be proportional to $B^{1 / 2}$ and $E^{-1 / 2}$ if the kinetic energy release is relatively large $\left(k_{i} \ll k_{f}\right)$. This is consistent with our results obtained from full quantum scattering calculations in the ultracold regime. As the collision energy increases, however, the assumption of $k_{i} \ll k_{f}$ breaks down and the cross sections deviate from the $B^{1 / 2}$ behavior. We find numerically that this is the case for energies above $\sim 10^{-6} \mathrm{~K}$ at nearly all the field strengths considered in this work (see Fig. 3). In a separate publication, we will give a general analytical expression for the inelastic cross section due to $V_{\text {magn.dip }}$ based on the (distorted-wave) Born approximation and show that the numerical and analytical results are in excellent agreement over a wide range of energies and fields.

At collision energies above about $10 \mathrm{mK}$ or at high magnetic fields, there is sufficient energy to overcome the $d$-wave centrifugal barrier in the outgoing channels and, as a consequence, short-range effects become important.
In particular the intramolecular spin-spin coupling term, which requires strong anisotropy of the potential in order to induce Zeeman relaxation [16,36], enhances the inelastic cross section significantly at energies above $\sim 10 \mathrm{mK}$. For the intramolecular contributions, we find that the inelastic cross section behaves as $B^{5 / 2}$ at moderate field strengths and flattens off to a constant value at very small $B$. Its kinetic energy dependence is proportional to $E^{-1 / 2}$ in the ultracold regime and, for small magnetic fields, also shows a region of $E^{2}$ behavior. This result is consistent with the work of Volpi and Bohn, who applied the distorted-wave Born approximation to calculate inelastic spin-changing collisions induced at short range [43]:

$$
\sigma_{i \rightarrow f} \propto E^{L_{i}-1 / 2}\left(E+g_{S} \mu_{\mathrm{B}} B \Delta M_{S}\right)^{L_{f}+1 / 2} .
$$

Thus, for an $s$-wave incoming channel $\left(L_{i}=0\right)$ and a $d$-wave outgoing channel $\left(L_{f}=2\right)$, there is a region of $E^{2}$ dependence when the Zeeman shift for the outgoing channel is small compared to the collision energy. At very small fields, the magnetic field dependence is negligible and the cross section becomes constant as a function of $B$.

We also point out that, in contrast to the intermolecular spin-spin coupling term, the intramolecular spin-spin interaction contains second-rank tensors in $\hat{\boldsymbol{S}}_{A}$ and $\hat{\boldsymbol{S}}_{B}$ and therefore directly couples channels where $M_{S_{A}}$ and $M_{S_{B}}$ each differ by 0,1 , or 2 . Thus, transitions from $M_{S_{i}}=1$ to $M_{S_{i}}=-1$ also become allowed in first order. This is illustrated in Fig. 6, where the state-to-state inelastic cross sections for $\mathcal{M}=2$ are plotted as a function of energy. In the ultracold region, which is dominated by $V_{\text {magn.dip }}$, only the $\left|M_{S_{A}}=1, M_{S_{B}}=1\right\rangle \rightarrow\left|M_{S_{A}}=1, M_{S_{B}}=0\right\rangle$ and $\mid M_{S_{A}}=$ $\left.1, M_{S_{B}}=1\right\rangle \rightarrow\left|M_{S_{A}}=0, M_{S_{B}}=0\right\rangle$ transitions contribute to the inelastic cross section. At higher energies, where the intramolecular spin-spin term plays a significant role, transitions from the $\left|M_{S_{A}}=1, M_{S_{B}}=1, L=0\right\rangle$ channel to the $\left|M_{S_{A}}=1, M_{S_{A}}=-1, L=2\right\rangle,\left|M_{S_{A}}=0, M_{S_{A}}=-1, L=4\right\rangle$, and $\left|M_{S_{A}}=-1, M_{S_{A}}=-1, L=4\right\rangle$ channels become increasingly important. Note that the latter two have $g$-wave barriers in the exit channel, and hence they are strongly suppressed in the low-energy regime.

In summary, we have established that the dominant trap-loss mechanism for $\mathrm{NH}$ in the ultracold regime is the intermolecular spin-spin coupling term, which induces Zeeman relaxation at long range. When the kinetic energy in the outgoing channel becomes large, the spin-change is also caused by the interplay of the potential anisotropy and the intramolecular spin-spin interaction, which acts at short range.

\section{Dependence on potential and basis-set size}

As a final part of our discussion, we consider the sensitivity to the potential and the dependence on the size of the channel basis set. Previous theoretical work on field-free collisions [32] has shown that the $\mathrm{NH}+\mathrm{NH}$ cross sections at low collision energies are very sensitive to the details of the potential-energy surface, which is due to the presence of quasibound state resonances. Since the exact form of the interaction potential, and thus the precise locations of the quasibound states, is still unknown, the calculated cross sections are subject to an inherent degree of uncertainty. The work of Ref. [32] 


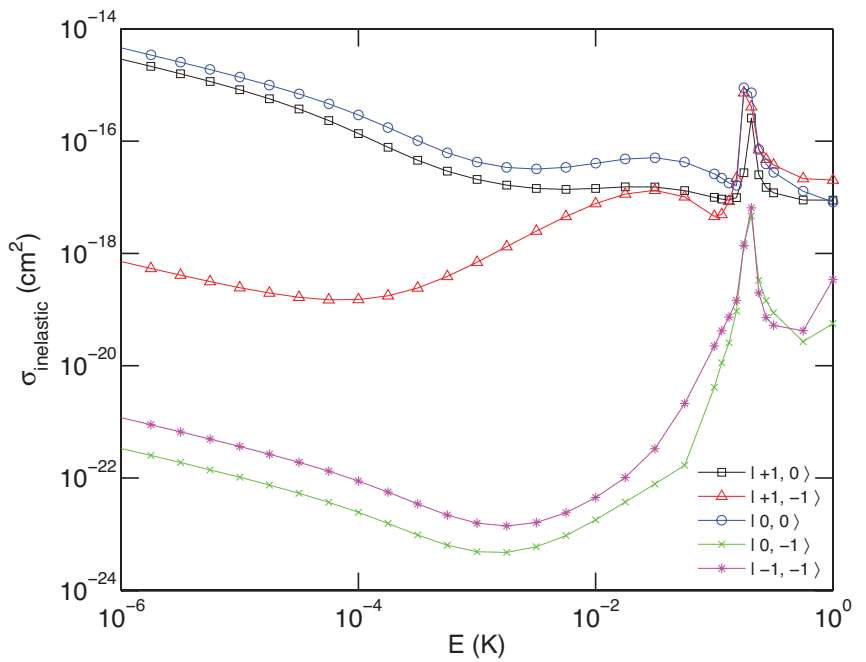

FIG. 6. (Color online) State-to-state inelastic cross sections $(\mathcal{M}=2)$ for magnetically trapped ${ }^{15} \mathrm{NH}$ as a function of energy at $1 \mathrm{G}$. The final states are labeled by $\left|M_{J_{A}}, M_{J_{B}}\right\rangle$.

showed, however, that the qualitative results and conclusions are reasonably independent of the details of the potential. Furthermore, it was demonstrated that the effect of using an unconverged channel basis set is very similar to a scaling of the potential within its uncertainty. That is, full basisset convergence is not strictly required in order to obtain qualitatively meaningful results [32].

Our present work is based on a similar approach. Following Refs. [24] and [32], we have studied the potential dependence indirectly by evaluating the sensitivity to the reduced mass. The reduced mass was scaled by a factor of $\lambda(0.9 \leqslant \lambda \leqslant 1.1)$, which is essentially equivalent to scaling of the entire potential by $\lambda$ [24]. Figure 7 shows the $\mathcal{M}=2$ cross sections for different scaling factors at a collision energy of $10^{-6} \mathrm{~K}$ and a field strength of $1 \mathrm{G}$. We find that the cross sections

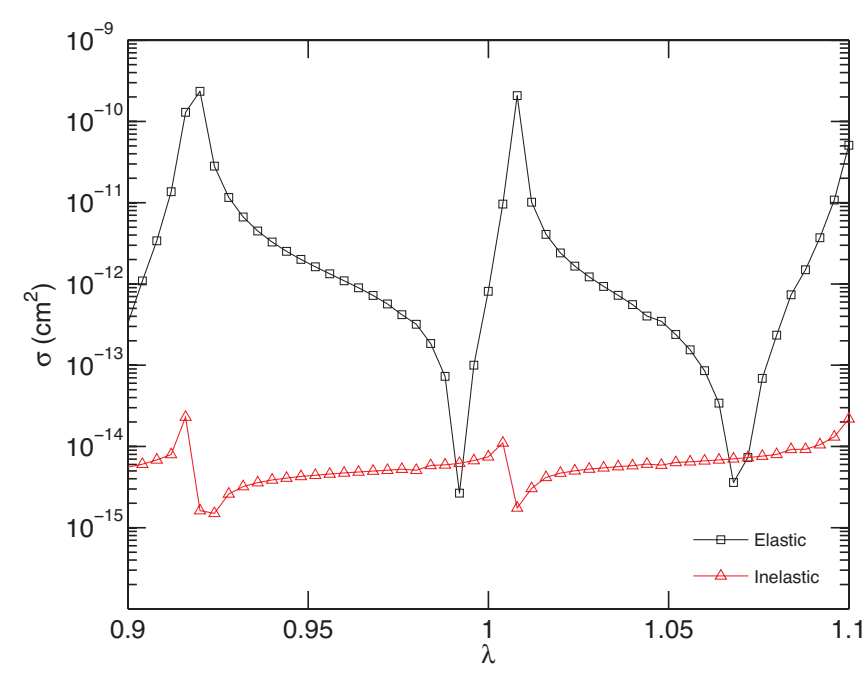

FIG. 7. (Color online) Elastic and inelastic $M_{J}$-changing cross sections $(\mathcal{M}=2)$ for magnetically trapped ${ }^{15} \mathrm{NH}$ as a function of the scaling factor $\lambda$, calculated at a collision energy of $10^{-6} \mathrm{~K}$ and a magnetic field strength of $1 \mathrm{G}$.

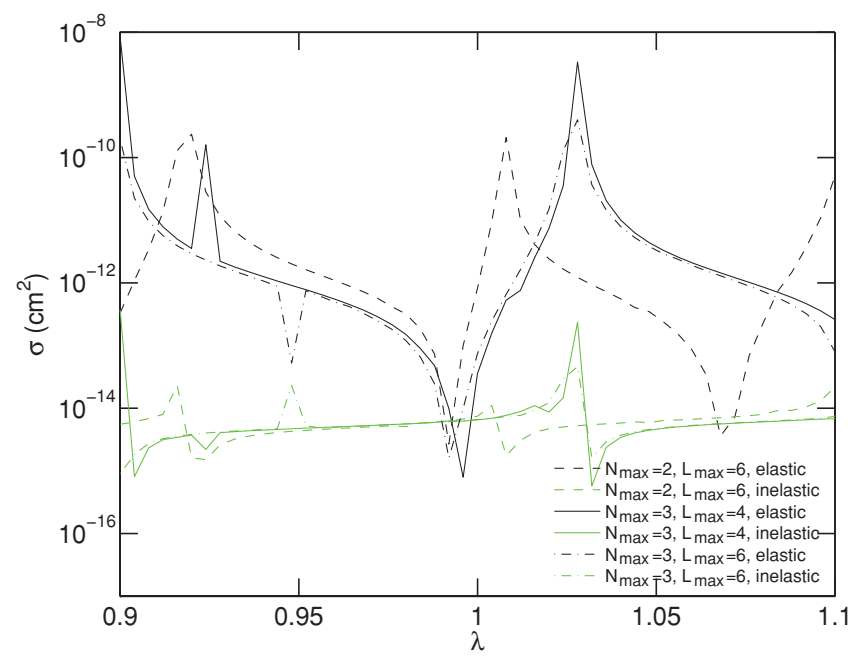

FIG. 8. (Color online) Elastic and inelastic $M_{J}$-changing cross sections $(\mathcal{M}=2)$ as a function of the scaling factor $\lambda$, calculated for different basis sets at a collision energy of $10^{-6} \mathrm{~K}$ and a magnetic field strength of $1 \mathrm{G}$.

exhibit resonance structures at certain $\lambda$ values, indicating a high sensitivity to the potential. However, the ratio between elastic and inelastic cross sections is much less sensitive to the reduced mass, and the prospects for evaporative cooling remain very positive over almost the entire range of $\lambda$. The contributions from the different spin-changing mechanisms, as described in the previous section, are also very similar for different reduced masses. Our qualitative results and conclusions are thus reasonably independent of the precise form of the potential.

We use the same scaling method to investigate the dependence on basis-set size. Figure 8 shows the $\mathcal{M}=2$ cross sections as a function of $\lambda$ for different values of $N_{\max }$ and $L_{\max }$ at $E=10^{-6} \mathrm{~K}$ and $B=1 \mathrm{G}$. The total number of channels in these calculations ranged from 901 for $N_{\max }=2$ and $L_{\max }=6$ up to 2621 for $N_{\max }=3$ and $L_{\max }=6$. It can be seen that the positions of the resonances shift when the basis size is increased, but the general pattern remains essentially the same. This is consistent with other work on $\mathrm{NH}$ [23,24,32]. We thus conclude that, within the uncertainty limits of $\lambda$, our qualitative results are not very sensitive to the size of the angular basis set.

Figure 8 also demonstrates that the cross sections are not yet converged with respect to $N_{\max }$ and $L_{\max }$. In fact, field-free $\mathrm{NH}-\mathrm{NH}$ calculations suggest that the basis set should extend to at least $N_{\max }=6$ and $L_{\max }=7$ to achieve full convergence [32], which would amount to a maximum of 25598 channels for $\mathcal{M}=0$ in the present decoupled basis set. Such calculations are highly infeasible with the currently available computer power. However, taking into account the uncertainty in the potential and the pronounced resonance structure, even a fully converged basis set would not give really reliable numerical values. Nonetheless, it must be emphasized that unconverged basis sets can give qualitatively reliable results. As already discussed in Refs. [32] and [23], full basis-set convergence is therefore not strictly necessary within the uncertainty limits of the potential. 


\section{CONCLUSIONS}

We have carried out full quantum scattering calculations to study cold and ultracold ${ }^{15} \mathrm{NH}-{ }^{15} \mathrm{NH}$ collisions in magnetic fields. The elastic and spin-changing cross sections for magnetically trapped $\mathrm{NH}$ are found to be very favorable for efficient evaporative cooling. We have identified the intermolecular spin-spin coupling term as the main trap-loss mechanism at low energies and small magnetic fields, while the intramolecular spin-spin term becomes increasingly important at higher energies and fields. The effect of spin-rotation coupling is almost negligible.

Finally, we have demonstrated that the numerical values of the cross sections are very sensitive to the details of the potential, but the qualitative results and conclusions are almost independent of the exact form of the surface. The size of the angular basis set, which is almost impossible to converge for systems such as $\mathrm{NH}-\mathrm{NH}$, does not significantly alter the results within the uncertainty limits of the potential. This inherent uncertainty in the calculated cross sections, however, clearly highlights the need for reliable experimental data.

\section{ACKNOWLEDGMENTS}

We gratefully acknowledge EPSRC for funding the collaborative project CoPoMol under the ESF EUROCORES programme EuroQUAM. L.M.C.J. and G.C.G. thank the Council for Chemical Sciences of the Netherlands Organization for Scientific Research (CW-NWO) for financial support.
[1] A. Micheli, G. K. Brennen, and P. Zoller, Nat. Phys. 2, 341 (2006).

[2] D. DeMille, Phys. Rev. Lett. 88, 067901 (2002).

[3] A. André, D. DeMille, J. M. Doyle, M. D. Lukin, S. E. Maxwell, P. Rabl, R. J. Schoelkopf, and P. Zoller, Nat. Phys. 2, 636 (2006).

[4] B. L. Lev, E. R. Meyer, E. R. Hudson, B. C. Sawyer, J. L. Bohn, and J. Ye, Phys. Rev. A 74, 061402 (2006).

[5] H. L. Bethlem and W. Ubachs, Faraday Discuss. 142, 25 (2009).

[6] M. R. Tarbutt, J. J. Hudson, B. E. Sauer, and E. A. Hinds, Faraday Discuss. 142, 37 (2009).

[7] S. Y. T. van de Meerakker, N. Vanhaecke, M. P. J. van der Loo, G. C. Groenenboom, and G. Meijer, Phys. Rev. Lett. 95, 013003 (2005).

[8] J. J. Gilijamse, S. Hoekstra, S. Y. T. van de Meerakker, G. C. Groenenboom, and G. Meijer, Science 313, 1617 (2006).

[9] J. J. Gilijamse, S. Hoekstra, S. A. Meek, M. Metsälä, S. Y. T. van de Meerakker, G. Meijer, and G. C. Groenenboom, J. Chem. Phys. 127, 221102 (2007).

[10] W. C. Campbell, G. C. Groenenboom, H.-I Lu, E. Tsikata, and J. M. Doyle, Phys. Rev. Lett. 100, 083003 (2008).

[11] R. V. Krems, PhysChemChemPhys 10, 4079 (2008).

[12] K. M. Jones, E. Tiesinga, P. D. Lett, and P. S. Julienne, Rev. Mod. Phys. 78, 483 (2006).

[13] T. Köhler, K. Góral, and P. S. Julienne, Rev. Mod. Phys. 78, 1311 (2006).

[14] J. D. Weinstein, R. deCarvalho, T. Guillet, B. Friedrich, and J. M. Doyle, Nature (London) 395, 148 (1998).

[15] H. L. Bethlem and G. Meijer, Int. Rev. Phys. Chem. 22, 73 (2003).

[16] R. V. Krems, H. R. Sadeghpour, A. Dalgarno, D. Zgid, J. Kłos, and G. Chałasiński, Phys. Rev. A 68, 051401 (2003).

[17] W. C. Campbell, E. Tsikata, H.-I Lu, L. D. van Buuren, and J. M. Doyle, Phys. Rev. Lett. 98, 213001 (2007).

[18] M. T. Hummon, W. C. Campbell, H.-I Lu, E. Tsikata, Y. Wang, and J. M. Doyle, Phys. Rev. A 78, 050702 (2008).

[19] S. Hoekstra, M. Metsälä, P. C. Zieger, L. Scharfenberg, J. J. Gilijamse, G. Meijer, and S. Y. T. van de Meerakker, Phys. Rev. A 76, 063408 (2007).

[20] S. Y. T. van de Meerakker, R. T. Jongma, H. L. Bethlem, and G. Meijer, Phys. Rev. A 64, 041401 (2001).
[21] P. Soldán, P. S. Żuchowski, and J. M. Hutson, Faraday Discuss. 142, 191 (2009).

[22] A. O. G. Wallis and J. M. Hutson, Phys. Rev. Lett. 103, 183201 (2009).

[23] A. O. G. Wallis, E. J. J. Longdon, P. S. Żuchowski, and J. M. Hutson, e-print arXiv:1009.5505.

[24] P. S. Żuchowski and J. M. Hutson, PhysChemChemPhys 13, 3669 (2011).

[25] M. T. Hummon, T. V. Tscherbul, J. Kłos, H.-I Lu, E. Tsikata, W. C. Campbell, A. Dalgarno, and J. M. Doyle, Phys. Rev. Lett. 106, 053201 (2011).

[26] W. Ketterle and N. J. van Druten, Evaporative Cooling of Atoms, Advances in Atomic, Molecular, and Optical Physics, edited by B. Bederson and H. Walther (Academic press, San Diego, CA, 1996), Vol. 37, pp. 181-236.

[27] S. C. Doret, C. B. Connolly, W. Ketterle, and J. M. Doyle, Phys. Rev. Lett. 103, 103005 (2009).

[28] G. S. F. Dhont, J. H. van Lenthe, G. C. Groenenboom, and A. van der Avoird, J. Chem. Phys. 123, 184302 (2005).

[29] L. M. C. Janssen, G. C. Groenenboom, A. van der Avoird, P. S. Żuchowski, and R. Podeszwa, J. Chem. Phys. 131, 224314 (2009).

[30] C.-H. Lai, M.-D. Su, and S.-Y. Chu, J. Phys. Chem. A 107, 2700 (2003).

[31] M. Kajita, Phys. Rev. A 74, 032710 (2006).

[32] L. M. C. Janssen, P. S. Żuchowski, A. van der Avoird, J. M. Hutson, and G. C. Groenenboom, e-print arXiv:1012.0804.

[33] R. S. Ram and P. F. Bernath, J. Mol. Spectrosc. 260, 115 (2010)

[34] M. Mizushima, The Theory of Rotating Diatomic Molecules (Wiley, New York, 1975).

[35] S. Green, J. Chem. Phys. 62, 2271 (1975).

[36] R. V. Krems and A. Dalgarno, J. Chem. Phys. 120, 2296 (2004)

[37] T. V. Tscherbul, Y. V. Suleimanov, V. Aquilanti, and R. V. Krems, New J. Phys. 11, 055021 (2009).

[38] M. H. Alexander and D. E. Manolopoulos, J. Chem. Phys. 86, 2044 (1987).

[39] J. M. Hutson and S. Green, MOLSCAT computer code, version 14 (1994), distributed by Collaborative Computational Project No. 6 of the Engineering and Physical Sciences Research Council, UK. 
[40] M. L. González-Martínez and J. M. Hutson, Phys. Rev. A 75, 022702 (2007).

[41] J. M. Hutson, E. Tiesinga, and P. S. Julienne, Phys. Rev. A 78, 052703 (2008).
[42] H. Cybulski, R. V. Krems, H. R. Sadeghpour, A. Dalgarno, J. Kłos, G. C. Groenenboom, A. van der Avoird, D. Zgid, and G. Chałasiński, J. Chem. Phys. 122, 094307 (2005).

[43] A. Volpi and J. L. Bohn, Phys. Rev. A 65, 052712 (2002). 